\title{
The long-term neurodevelopmental outcomes of infants born full-term with low birth weight
}

\author{
Ayşe Tosun'1, Börte Gürbüz-Özgür², Hatice $\mathrm{Aksu}^{3}$, Münevver Kaynak-Türkmen ${ }^{4}$ \\ Departments of ${ }^{1}$ Pediatric Neurology, ${ }^{3}$ Child and Adolescent Psychiatry, and ${ }^{4}$ Neonatology, Adnan Menderes University \\ Faculty of Medicine, Aydın; ${ }^{2}$ Department of Child and Adolescent Psychiatry, Muğla Sıtkı Koçman University, Training \\ and Research Hospital, Muğla, Turkey. E-mail:aysetosun2000@yahoo.com \\ Received: 30th January 2017, Accepted: 11th February 2017
}

SUMMARY: Tosun A, Gürbüz-Özgür B, Aksu H, Kaynak-Türkmen M. The long-term neurodevelopmental outcomes of infants born full-term with low birth weight. Turk J Pediatr 2017; 59: 169-176.

In this study, it was aimed to evaluate the neurological developments of preschool or school-aged children together with their school successes, intelligence quotient and symptom severity of attention deficit hyperactivity disorder who were born at the $37^{\text {th }}$ gestational week and above with birth weights below tenth percentile, which is called small for gestational age (SGA). A total of 74 patients with SGA and 75 healthy children were evaluated. The patients were evaluated by child neurologist and child psychiatrist. Wechsler Intelligence Scale for Children-Revised, Ankara Developmental Screening Inventory, and The Turgay DSM-IV-Based Child and Adolescent Disruptive Behavioral Disorders Screening and Rating Scale (T-DSM-IV-S) were applied according to their age groups. SGA cases had been breastfed for shorter durations ( $p: 0.004)$, had walked later $(p<0.001)$, talked later $(p<0.001)$, and had encountered more vision disorders ( $p: 0.009)$ than the control group. SGA cases were determined to encounter febrile convulsions more frequently ( $\mathrm{p}$ : 0.007). SGA cases were determined to exhibit lower school success $(p<0.001)$, were diagnosed with attention deficit hyperactivity disorder more frequently $(\mathrm{p}<0.001)$, and their mental developments were delayed $(\mathrm{p}<0.001)$. In cases with SGA, inattention (p: 0.004) and conduct disorder ( $p: 0.029)$ subscales and the total scale scores (p: 0.022) of T-DSM-IV-S were significantly lower when compared to the control group. We consider that being SGA may have a negative impact on child's behavior, attention and academic achievement in long-term.

Key words: academic achievement, attention deficit hyperactivity disorder, behavior, children, small for gestational age.

The intrauterine life is the period in which the growth and development are in their fastest states. Fetal growth shows variations throughout the pregnancy. While the healthy fetus fully reaches the genetically determined somatic growth potential and functional maturation, the normal growth and development of the fetus are altered in the presence of any risk factor. These developmental stages may be affected by the maternal environment and uteroplacental functions, as well as the genetic potential of the fetus. The small for gestational age (SGA) term is defined as the birth weight being lower than ten percentile or two standard deviations (SD) according to the gestational week ${ }^{1-4}$. This situation affects $3-10 \%$ of all newborns ${ }^{5}$. SGA is classified as symmetrical and asymmetrical SGA, based on the ponderal index and the percentiles of height and head circumference. When the intrauterine growth retardation has been present since the early gestational period, the head (brain) size is proportional to the body growth, and the ponderal index is normal, it is considered as symmetrical SGA; if it is not proportional, asymmetrical SGA is considered. Placental insufficiency, maternal preeclampsia, hypertension, long-term presence of diabetes mellitus, smoking, living at high altitude, and the multiple pregnancies are among the etiologies of asymmetrical 
SGA. Congenital infections, chromosomal and other anomalies, fetal alcohol syndrome, low socioeconomic status, and constitutional causes take place in the etiology of symmetrical $\mathrm{SGA}^{4,6,7}$. Babies who are born with SGA have perinatal morbidity or perinatal mortality risks due to causes such as acidosis, hypoglycemia, hypocalcemia, hypothermia, polycythemia, coagulation disorders, susceptibility to infections, and necrotizing enterocolitis. These babies are confronted with risks such as hypertension, cardiovascular disorders, diabetes mellitus, metabolic syndrome, short stature, and neurological and developmental disorders in the long-term 5,8 . However, minor neurological dysfunctions such as attention deficit hyperactivity disorder (ADHD), academic underachievement, and clumsiness are seen more frequently than major neurological sequela such as cerebral palsy in cases with SGA. The studies have shown that nutrition is essential for brain development and affects the structural development of the brain; together with learning, memory, school success, and IQ, SAG led to behavioral and emotional problems ${ }^{8-12}$. Additionally, long-term adverse effects of SGA on the language and conversational development (delay in talking,

Table I. The Demographic Characteristics of the Case and Control Groups.

\begin{tabular}{|c|c|c|c|}
\hline & SGA (n: 74) & Control (n: 75) & $\mathrm{p}$ \\
\hline Age* (years) $^{*}$ & $9(7-11)$ & $8(8-10)$ & \\
\hline Gender $(\mathrm{F} / \mathrm{M}), \mathrm{n} / \mathrm{n}$ & $44 / 30$ & $43 / 32$ & \\
\hline Gestational age (weeks) & $38.9 \pm 0.6$ & $38.5 \pm 0.5$ & \\
\hline Birth weight* (gr) & $2,275(2,000-2,480)$ & $3,250(3,000-3,500)$ & \\
\hline $\begin{array}{l}\text { Mode of delivery, n (\%) } \\
\text { NSpV } \\
\text { C/S }\end{array}$ & $\begin{array}{l}47 \\
27\end{array}$ & $\begin{array}{l}56 \\
19\end{array}$ & 0.195 \\
\hline $\begin{array}{l}\text { Acidosis/asphyxia, n (\%) } \\
\text { Present } \\
\text { Absent }\end{array}$ & $\begin{array}{l}8(10.8) \\
66(89.2)\end{array}$ & $\begin{array}{l}0 \\
75(100.0)\end{array}$ & 0.003 \\
\hline $\begin{array}{l}\text { Follow-up in the incubator, } \mathrm{n}(\%) \\
\text { Present } \\
\text { Absent } \\
\text { Maternal age* (years) }\end{array}$ & $\begin{array}{l}17(23.0) \\
57(77.0) \\
26(21.5-29)\end{array}$ & $\begin{array}{ll}1 & (1.4) \\
73 & (98.6) \\
26 & (21-29)\end{array}$ & $\begin{array}{l}<0.001 \\
0.696\end{array}$ \\
\hline Number of pregnancies* & $2(2-3)$ & $2(2-3)$ & 0.144 \\
\hline $\begin{array}{l}\text { Mother working status, n (\%) } \\
\text { Housewife } \\
\text { Working }\end{array}$ & $\begin{array}{ll}54 & (73.0) \\
20 & (27.0)\end{array}$ & $\begin{array}{ll}62 & (82.7) \\
13 & (17.3)\end{array}$ & 0.22 \\
\hline $\begin{array}{l}\text { Educational status of the mother, } \mathrm{n}(\%) \\
\text { Illiterate } \\
\text { Literate } \\
\text { Primary school } \\
\text { Secondary education } \\
\text { University } \\
\text { Father's age }\end{array}$ & $\begin{array}{l}12(16.7) \\
5(6.9) \\
41(56.9) \\
8(11.2) \\
6(8.3) \\
40(35-43)\end{array}$ & $\begin{array}{l}5(6.9) \\
0 \\
40(53.3) \\
23(30.6) \\
7(9.3) \\
38.5(35-44)\end{array}$ & 0.009 \\
\hline $\begin{array}{l}\text { Educational status of the father, } \mathrm{n}(\%) \\
\text { Illiterate } \\
\text { Literate } \\
\text { Primary school } \\
\text { Secondary education } \\
\text { University }\end{array}$ & $\begin{array}{l}3(4.2) \\
2(2.8) \\
42(59.2) \\
19(26.8) \\
5(7.0)\end{array}$ & $\begin{array}{l}1(1.3) \\
0 \\
45(60.0) \\
25(33.4) \\
4(5.3)\end{array}$ & 0.58 \\
\hline $\begin{array}{l}\text { Parental consanguinity, n (\%) } \\
\text { Present } \\
\text { Absent }\end{array}$ & $\begin{array}{l}14(18.9) \\
60(81.1)\end{array}$ & $\begin{array}{l}8(10.7) \\
67(89.3)\end{array}$ & 0.235 \\
\hline Presence of psychiatric disorder in parents, $\mathrm{n}(\%)$ & $12(16.2)$ & $10(13.3)$ & 0.791 \\
\hline
\end{tabular}

*: data is presented as median $\left(25^{\text {th }}-75^{\text {th }}\right.$ percentile); NSpV: normal spontaneous vaginal birth; C/S: Cesarean section 
Table II. Developmental Steps and Frequency of Disorders in the SGA and Control Groups.

\begin{tabular}{llll}
\hline Developmental steps and disorders & SGA (n: 74) & Control (n: 75) & $\mathrm{p}$ \\
\hline Walking* (months) & $13(12-18)$ & $12(12-13)$ & $<0.001$ \\
Talking* (months) & $12(12-24)$ & $12(10-12)$ & $<0.001$ \\
Toilet training* (years) & $2(2-3)$ & $2(2-2.5)$ & 0.33 \\
Febrile convulsions, n (\%) & & & 0.007 \\
$\quad$ Present & $11(14.9)$ & $1(1.4)$ & \\
Absent & $63(85.1)$ & $73(98.6)$ & 0.058 \\
Epilepsy, n (\%) & $5(6.8)$ & 0 & \\
Present & $69(93.2)$ & 0 & 0.009 \\
Absent & $9(12.2)$ & $1(1.3)$ & \\
Vision disorders, n (\%) & $65(87.8)$ & $74(98.7)$ & \\
$\quad$ Present & $7(9.5)$ & 0 & \\
Absent & $67(90.5)$ & 0 & \\
Special education, n (\%) & & & \\
$\quad$ Present & & & \\
Absent &
\end{tabular}

*: data is presented as median $\left(25^{\text {th }}-75^{\text {th }}\right.$ percentile)

poor and immature language and articulation disorders), visual-motor integration, fine and gross motor skills have been reported $11,13,14$.

Sufficient amount of studies showing the longterm neurodevelopmental outcomes of SGA cases is not present. In this study, we aimed to evaluate the neurodevelopmental status of full-term babies with SGA in their pre-school and school ages.

\section{Material and Methods}

The approval of University Ethics Committee and the written consents of parents were obtained. The study was in accordance with the Declaration of Helsinki for human subjects.

All cases, between 6-16 years of age, who had been born in Medical Faculty Hospital with gestational ages of 37 weeks and over, and with the diagnosis of SGA were included in the study. First, the prenatal-perinatal risk factors concerning the neonatal period of the cases were retrospectively recorded through chart review in this study. They were evaluated by the same child neurologist and child psychiatrist, respectively. Wechsler Intelligence Scale for Children-Revised Intelligence Test (WISC-R) ${ }^{15}$, Ankara Developmental Screening Inventory (ADSI) ${ }^{16}$, and the Turgay DSMIV-Based Child and Adolescent Disruptive Behavioral Disorders Screening and Rating Scale (T-DSM-IV-S) ${ }^{17}, 18$ were applied according to their age groups. Healthy children, born over $37^{\text {th }}$ gestational week with birth weight appropriate for gestational age (AGA), who were admitted to the Pediatric Outpatient Clinic for any reason, were included in the control group. In the control group, mental development was evaluated according to the American Psychiatric Association's Mental Disorders Diagnostic and Statistical Manual revised $4^{\text {th }}$ version (DSM-IV-TR) diagnostic criteria $^{19}$ and their parents filled out the T-DSM-IV-S. School achievement was evaluated according to information gathered from child's school teacher in both SGA and control groups. Infants born at less than 37 weeks of gestational age, having a genetic syndrome, major malformation, or congenital infection, diagnosed with a neurometabolic disorder, and having medical histories of diseases that can adversely affect the neurodevelopment, such as intracranial bleeding, sepsis, and hyperbilirubinemia requiring blood exchange transfusion were excluded from the study.

\section{Performed Tests}

Wechsler Intelligence Scale for Children-Revised Intelligence Test (WISC-R): It is applied to individuals aged 6-16 years. The test consists of 12 subtests involving verbal (general knowledge ability, similarities, arithmetic, vocabulary, reasoning, digit span) and performance (picture completion, image reorganization, designing by using cubes, joining the pieces, password, labyrinths) skills. The total test score is determined by summing the verbal and performance scores. Charts are used for determination of verbal, performance, and total 
Table III. The School Success, Attention Deficit Hyperactivity Disorder Diagnosis, and Results of the Intelligence Level According to SGA and Control Groups.

\begin{tabular}{llll}
\hline Features & SGA Group & Control Group & $\mathrm{p}$ \\
\hline School success, n (\%) & $36(52.2)$ & $68(90.7)$ & $<0.001$ \\
$\quad$ Normal & $33(47.8)$ & $7(9.3)$ & \\
$\quad$ Low & $27(36.5)$ & $8(10.7)$ & $<0.001$ \\
ADHD, n (\%) & $47(63.5)$ & $67(89.3)$ & \\
$\quad$ Present & & $70(93.3)$ & $<0.001$ \\
$\quad$ Absent & $47(63.5)$ & $5(6.7)$ & \\
Intelligence level, n (\%) & $14(18.9)$ & 0 & \\
$\quad$ Normal & $8(10.8)$ & 0 & \\
$\quad$ Borderline intelligence & $4(5.4)$ & 0 & \\
$\quad$ Mild mental retardation & $1(1.4)$ & & \\
Moderate mental retardation & & \\
Severe mental retardation & &
\end{tabular}

ADHD: Attention Deficit Hyperactivity Disorder

intelligence sections.

Ankara Developmental Screening Inventory (ADSI): It is an inventory used for evaluation of developmental aspects of children aged between 0-6 years. Speech development, cognitive skills, fine muscle development, gross muscle development, social development, and selfcare skills are observed and scored. ADSI was applied to children who cannot get WISC-R.

The Turgay DSM-IV-Based Child and Adolescent Disruptive Behavioral Disorders Screening and Rating Scale (T-DSM-IV-S): It is a scale for screening the disruptive behavioral disorders, which was developed by Turgay and its Turkish validity-reliability study was conducted by Ercan et al.17,18. A total of 41 questions in the scale have items questioning attention deficit, hyperactivity/impulsivity, oppositional defiant disorder and behavior disorder. The severity of each item is measured by four-point Likerttype scoring.

\section{Statistical Analysis}

The statistical software package SPSS Statistics for Windows Version 17 (SPSS Inc., Chicago, IL, USA) was used for all the statistical analyses. The conformity of quantitative variables with normal distribution was analyzed by Kolmogorov-Smirnov test. Since the qualitative variables did not have a normal distribution, Mann-Whitney U-test was used for comparison of two groups and descriptive statistics were shown as median $\left(25^{\text {th }}-75^{\text {th }}\right.$ percentiles). Chi-square test was used for comparison of qualitative variables, and descriptive statistics were displayed as frequency (\%); $<<0.05$ was considered as statistically significant.

\section{Results}

Seventy-four cases with SGA and 75 healthy children, constituting the control group, were included in the study. The demographic data of cases with SGA and the control group were shown in Table I. No statistically significant difference was found between the cases with SGA and the control group in terms of maternal age at birth (p: 0.696), father's age (p: 0.696), the total number of pregnancies of the mother (p: 0.144), and causes which may lead to SGA, such as maternal gestational infection, hypertension, cardiac disorder, renal disorder, diabetes mellitus, and multiple pregnancies $(p>0.05)$. The educational level of the mother was significantly lower in cases with SGA (p: 0.009) than the control group. No statistically significant difference was found between cases with SGA and the control group regarding complications such as hypoglycemia, hypocalcemia, hypothermia, and NEC, which may develop related to SGA, following birth. However, in cases with SGA, duration of stay in the incubator $(\mathrm{p}<0.001)$ and asphyxia rate (p: 0.003) were significantly higher in the cases with SGA, when compared to the control group (Table I).

The cases with SGA were breastfed for shorter durations ( $p$ : 0.004), walked and talked later $(p<0.001)$, vision disorders more frequently ( $p$ : 0.009 ) and received mental special educational support (p: 0.006) compared to the control group. Hearing loss was detected in two 
cases. In the cases with SGA were determined febrile convulsions more frequently (p: 0.007), but epilepsy was not significant difference between the groups (two SGA cases were diagnosed with epilepsy) (p: 0.058). However, physical therapy and rehabilitation support, and chronic disorders (chronic pulmonary, renal, cardiovascular problems, hypertension, obesity, diabetes mellitus), no significant difference was found between the groups (Table II).

The school success was found as significantly lower in the cases with SGA $(p<0.001)$; however, no difference was present between genders. The incidence of ADHD was significantly higher in the cases with SGA $(p<0.001)$, and higher in male gender when compared to females (p: 0.007). In the SGA group, ADSI was applied in 12 and WISC-R was implemented in 61 cases. According to the results of ADSI, in 2 (16.6\%) cases moderate-level mental retardation, in 3 $(25 \%)$ cases mild-level mental retardation, in 2 $(16.6 \%)$ cases borderline mental functionality, and in $5(41.7 \%)$ cases, normal psychomotor development was determined. According to the results of WISC-R, applied in the 61 cases with SGA, $63.5 \%$ had normal (IQ: 90-110), and $18.9 \%$ had borderline (IQ: $70-$ 90) intelligence; $10.8 \%$ had mild mental retardation (IQ: 50-69), 5.4\% had moderate mental retardation (IQ: $35-49$ ), and $1.4 \%$ had severe mental retardation (IQ: 20-34). In the control group, $93.3 \%$ had normal, and $6.7 \%$ had borderline intelligence $(p<0.001)$. There was no significant difference between genders regarding intelligence levels. School success, presence of ADHD, and intelligence levels of the cases with SGA and the control group were shown in Table III.

T-DSM-IV-S, which investigates the symptoms related to behaviors and attention, was applied to the parents. The scores related to the attention deficit subscale (p: 0.004), conduct disorder subscale (p: 0.029), and parental evaluation scale (p: 0.022) were found significantly higher compared to the control group. However, no difference was determined between genders. Statistically significant difference was not found between groups regarding the hyperactivity/ impulsiveness and oppositional defiant disorder subscales. T-DSM-IV-S scores of the SGA and control groups were given in Table IV.

\section{Discussion}

The intrauterine life is the period in which the growth and development are in their fastest states. Fetal growth is affected by the presence of any risk factor. Studies have shown that, in babies born with SGA, there are mild neurological, behavioral, emotional problems at various levels, and their cognitive scores and academic successes are lower when compared to children born with a normal birth weight according to gestational age. When the literature was analyzed, low birth weight was found to be related to problems such as attention deficit, ADHD, anxiety-depression, aggression, and disorders of mental state involving symptoms of both introversion and extroversion ${ }^{20-24}$.

Jeliffe-Pawlowski LL, et al. ${ }^{25}$ evaluated the term SGA babies by Bayley Developmental Test in the $8^{\text {th }}$ month and Stanford Binet Intelligence Test in the $4^{\text {th }}$ age. They stated that neurodevelopmental retardation became more evident as the age progressed, this increase being more significant in the low socio-economic group when compared to the intermediate-high socio-economic group. They considered that this difference might have originated from biological, economic, and/or familial factors. In our study, the significantly lower educational level of mothers of SGA

Table IV. The Comparison of Parental T-DSM-IV-S Scores of the SGA and Control Groups.

\begin{tabular}{llll}
\hline T-DSM-IV-S subscale types & SGA $(\mathrm{n}=54)$ & Control $(\mathrm{n}=75)$ & $\mathrm{p}$ \\
\hline Attention deficit & $7.5(3-15.25)$ & $4(2-7)$ & 0.004 \\
Hyperactivity-impulsivity & $4(1-11.25)$ & $4(2-7)$ & 0.922 \\
Oppositional defiant disorder & $3(1-8.25)$ & $4(1-7)$ & 0.939 \\
Conduct disorder & $0.0(0.0-2)$ & $0.0(0.0-1)$ & 0.029 \\
Total & $17(10-31.5)$ & $12(7-23)$ & 0.022
\end{tabular}

T-DSM-IV-S: the Turgay DSM-IV-Based Child and Adolescent Disruptive Behavioral Disorders Screening and Rating Scale. Data is presented as median $\left(25^{\text {th- }}-75^{\text {th }}\right.$ percentile) 
cases when compared to the control group (p: 0.009 ), their increased number of pregnancies $(p \geq 0.05)$, and the higher incidence of unskilled jobs among them ( $27 \%$ vs. $17 \%, \mathrm{p} \geq 0.05)$ gave rise to the thought that, although not statistically significant, these mothers were in the low socio-economic income group.

In our study, the cases with SGA were determined to encounter asphyxia more frequently (p: 0.003) and stayed in the incubator for a longer duration $(\mathrm{p}<0.001)$ when compared to the control group. This situation may explain the probable existence of stage I hypoxic ischemic encephalopathy in cases with SGA and why the cases with SGA stayed for longer duration in the incubator concerning the follow-up for the development of postdelivery complications such as hypothermia, hypoglycemia, polycythemia, and coagulation disorders.

In the literature, the existence of the major neurological handicaps in cases with SGA has been quite rarely reported $21,24,26$. In our study, no case diagnosed with cerebral palsy as in the literature was present; however, similar to the literature $^{14}$ these cases were more delayed in walk and talk than the control group. It was also reported that minor neurological dysfunction was seen more frequently, whereas vision and hearing disorders constituted fewer problems in cases with SGA. The refractive errors were reported to be more frequent in cases with SGA, particularly hypermetropia in girls 27,28 . In our study, vision disorders were more prevalent in the cases with SGA, and they used eyeglasses when compared to the control group. On the other hand, no significant differences were found between groups concerning hearing problems and the period of gaining control in toilet training.

Visser et al. ${ }^{29}$ reported that the febrile convulsion risk was increased within the first two years of age in cases having fetal growth retardation. Moreover, Vestergaard et al. ${ }^{30}$ determined an increased febrile convulsion risk in cases with low birth weight. In the present study, in cases with SGA, febrile convulsion frequency was higher than the control group (p: 0.007), but there was no significant difference between the groups, regarding the development of epilepsy.

Arcangeli et al. ${ }^{3}$ reported that the neurodevelopmental scores were lower in fullterm babies with SGA than full-term babies with AGA in a meta-analysis study. They reported that the standardized neurodevelopmental scores were 0.32 SD less than normal controls. In another study, a $10-15 \%$ increase in schoolrelated problems was identified in cases with SGA. In studies with long-term follow-up, the probability of low educational level was found $33 \%$ higher in cases with SGA ${ }^{24}$. Leitner et al., 5 in children with SGA, showed the existence of cognitive problems such as minor neurodevelopmental problems, learning difficulties, memory performance, visuomotor functions, and short attention span, together with lower levels of IQ and school success. In a study by O'Keefe et al. ${ }^{31}$ learning difficulties, and particularly in girls attention deficits, were more frequent in children with SGA; however, there was no difference between the IQs. Low JA et al. ${ }^{23}$ reported that learning difficulties were present in $35 \%$ cases, and lower IQ scores, behavioral disorders, inattention, and anxiety issues in these children. In another study, $10^{\text {th }}$ age academic success was lower in SGA cases, but there was no significant difference between them in terms of WISC-R results ${ }^{22}$. Zubrick SR et al. ${ }^{20}$ stated that a wider range of mental health problems was present (anxiety, depression, ADHD, and conduct disorder) in cases with low birth weights. In our study, similar to the literature $5,22,24,32$, the school success was found to be lower in the cases with SGA when compared to the control group $(47.8 \%$ vs. $9.3 \%)$, and they were diagnosed with ADHD more frequently.

When the inattention and conduct disorder scores in the parent form of T-DSM-IV-S, which evaluates the symptom severity of attention deficit-hyperactivity disorder and conduct disorder, were compared to the control group, an increase was observed in the SGA cases, but there was no significant difference in terms of impulsiveness and oppositional defiant disorder scores. The total score of the parent form was higher in the cases with SGA. We considered that this might have originated from accompanying psychological problems due to the mental retardation frequently present in varying degrees in SGA cases.

\section{Limitations of the study}

The discrimination of asymmetrical - symmetrical 
SGA was not possible due to deficiencies in recording the height and head circumference measurements in SGA cases at birth.

Due to the lack of data concerning weight, height, and head circumference measurements in cases with SGA and the control group, the anthropometric data of SGA cases could not be evaluated.

The intelligence level of the control group was not determined by psychometric measurements.

In conclusion, we consider that being SGA (among babies born at term) may have a negative impact on child's behavior, attention and academic achievement in long-term.

\section{Acknowledgement}

The project was supported by Adnan Menderes University Department of Scientific Research Projects (Project Nr: 13022).

\section{REFERENCES}

1. Maulik D. Fetal growth compromise: definitions, standards, and classification. Clin Obstet Gynecol 2006; 49: 214-218.

2. Lee PA, Houk CP. Cognitive and psychosocial development concerns in children born small for gestational age. Pediatr Endocrinol Rev 2012; 10: 209-216.

3. Arcangeli T, Thilaganathan B, Hooper R, Khan KS, Bhide A. Neurodevelopmental delay in small babies at term: A systematic review. Ultrasound Obstet Gynecol 2012; 40: 267-275.

4. Halliday HL. Neonatal management and long-term sequelae. Best Pract Res Clin Obstet Gynaecol 2009; 23: $871-880$.

5. Leitner Y, Fattal-Valevski A, Geva R, et al. Neurodevelopmental outcome of children with intrauterine growth retardation: A longitudinal, 10-year prospective study. J Child Neurol 2007; 22: 580-587.

6. Maulik D. Fetal growth restriction: The etiology. Clin Obstet Gynecol 2006; 49: 228-235.

7. Neerhof MG, Thaete LG. The fetal response to chronic placental insufficiency. Semin Perinatol 2008; 32: 201205.

8. de Bie HM, Oostrom KJ, Delemarre-van de Waal HA. Brain development, intelligence and cognitive outcome in children born small for gestational age. Horm Res Paediatr 2010; 73: 6-14.

9. Fattal-Valevski A, Toledano-Alhadef H, Leitner Y, Geva R, Eshel R, Harel S. Growth patterns in children with intrauterine growth retardation and their correlation to neurocognitive development. J Child Neurol 2009; 24: $846-851$.
10. Jacobsson B, Ahlin K, Francis A, Hagberg G, Hagberg $\mathrm{H}$, Gardosi J. Cerebral palsy and restricted growth status at birth: population-based case-control study. BJOG 2008; 115: 1250-1255.

11. Frisk V, Amsel R, Whyte HE. The importance of head growth patterns in predicting the cognitive abilities and literacy skills of small-for-gestational-age children. Dev Neuropsychol 2002; 22: 565-593.

12. Pryor J, Silva PA, Brooke M. Growth, development and behaviour in adolescents born small-for-gestational-age. J Paediatr Child Health 1995; 31: 403-407.

13. Kisilevsky BS, Davies GA. Auditory processing deficits in growth restricted fetuses affect later language development. Med Hypotheses 2007; 68: 620-628.

14. Simic Klaric A, Kolundzic Z, Galic S, Mejaski Bosnjak V. Language development in preschool children born after asymmetrical intrauterine growth retardation. Eur J Paediatr Neurol 2012; 16: 132-137.

15. Savaşır I, Şahin, N. Wechsler Çocuklar İçin Zeka Ölçeği (WISCR) El Kitabı. Ankara: Türk Psikologlar Derneği Yayınları, 1995.

16. Savaşır N, Sezgin N, Erol N. Ankara Gelişim Tarama Envanteri El Kitabı (Genişletilmiş 3. Basım). Ankara: Rekmay, 2005.

17. Turgay A, Disruptive Behavior Disorders: Child and Adolescent Screening and Rating Scale for Children, Adolescents, Parents, and Teachers. West Blomfield, MI: Integrative Therapy Institute Publication, 1994.

18. Ercan ES, Amado S, Somer O, Çıkoğlu S. Development of a test battery for the assessment of attention deficit hyperactivity disorder. Turk J Child Adolesc Ment Health 2001; 8: 132-144.

19. American Psychiatric Association. Diagnostic criteria from DSM-IV-TR. Washington, DC: American Psychiatric Association, 2000: 370,xii.

20. Zubrick SR, Kurinczuk JJ, McDermott BM, McKelvey RS, Silburn SR, Davies LC. Fetal growth and subsequent mental health problems in children aged 4 to 13 years. Dev Med Child Neurol 2000; 42: 14-20.

21. Goldenberg RL, Hoffman HJ, Cliver SP. Neurodevelopmental outcome of small-for-gestationalage infants. Eur J Clin Nutr 1998; 52 (Suppl 1): S54-S58.

22. Hollo O, Rautava P, Korhonen T, Helenius H, Kero P, Sillanpaa M. Academic achievement of small-forgestational-age children at age 10 years. Arch Pediatr Adolesc Med 2002; 156: 179-187.

23. Low JA, Handley-Derry MH, Burke SO, Peters RD, Pater EA, Killen HL, et al. Association of intrauterine fetal growth retardation and learning deficits at age 9 to 11 years. Am J Obstet Gynecol 1992; 167: 1499-1505.

24. Shah P, Kingdom J. Long-term neurocognitive outcomes of SGA/IUGR infants. Obstet Gynaecol Reprod Med 2011; 21: 142-146.

25. Jelliffe-Pawlowski LL, Hansen RL. Neurodevelopmental outcome at 8 months and 4 years among infants born full-term small-for-gestational-age. J Perinatol 2004; 24: 505-514. 
26. Lundgren EM, Tuvemo T. Effects of being born small for gestational age on long-term intellectual performance. Best Pract Res Clin Endocrinol Metab 2008; 22: 477488.

27. Lindqvist S, Vik T, Indredavik MS, Brubakk AM. Visual acuity, contrast sensitivity, peripheral vision and refraction in low birthweight teenagers. Acta Ophthalmol Scand 2007; 85: 157-164.

28. Akova-Budak B, Kivanc SA, Olcaysu OO. Association of birth parameters with refractive status in a sample of caucasian children aged 4-17 years. J Ophthalmol 2015; 2015: 635682.

29. Visser AM, Jaddoe VW, Hofman A, Moll HA, Steegers EA, Tiemeier $\mathrm{H}$, et al. Fetal growth retardation and risk of febrile seizures. Pediatrics 2010; 126: e919-e925.
30. Vestergaard M, Basso O, Henriksen TB, Ostergaard JR, Olsen J. Risk factors for febrile convulsions. Epidemiology 2002; 13: 282-287.

31. O'Keeffe MJ, O'Callaghan M, Williams GM, Najman JM, Bor W. Learning, cognitive, and attentional problems in adolescents born small for gestational age. Pediatrics 2003; 112: 301-307.

32. Paz I, Gale R, Laor A, Danon YL, Stevenson DK, Seidman DS. The cognitive outcome of full-term small for gestational age infants at late adolescence. Obstet Gynecol 1995; 85: 452-456. 